\title{
Tension Space Analysis: Exploring Community Requirements for Networked Urban Screens
}

\author{
Steve North ${ }^{1}$, Holger Schnädelbach ${ }^{1}$, Ava Fatah gen Schieck ${ }^{2}$, Wallis Motta ${ }^{2}$, \\ Lei $\mathrm{Ye}^{1}$, Moritz Behrens ${ }^{2}$, and Efstathia Kostopoulou ${ }^{2}$ \\ ${ }^{1}$ Mixed Reality Laboratory (MRL), Department of Computer Science, \\ University of Nottingham, Nottingham, UK \\ \{Steve.North, Holger.Schnadelbach, L.Ye\} @nottingham.ac.uk \\ 2 The Bartlett, University College London, London, UK \\ \{ava.fatah, w.motta, moritz.behrens, ucftefk\}@ucl.ac.uk
}

\begin{abstract}
This paper draws on the design process, implementation and early evaluation results of an urban screens network to highlight the tensions that emerge at the boundary between the technical and social aspects of design. While public interactive screens in urban spaces are widely researched, the newly emerging networks of such screens present fresh challenges. Researchers wishing to be led by a diverse user community may find that the priorities of some users, directly oppose the wishes of others. Previous literature suggests such tensions can be handled by 'goal balancing', where all requirements are reduced down to one set of essential, implementable attributes. Contrasting this, this paper's contribution is 'Tension Space Analysis', which broadens and extends existing work on Design Tensions. It includes new domains, new representational methods and offers a view on how to best reflect conflicting community requirements in some aspects or features of the design.
\end{abstract}

Keywords: 'tension space analysis', 'human factors', 'design tensions', 'design space', 'urban screens', 'networked urban screens'.

\section{Introduction}

The contribution to knowledge presented by the authors is a development of existing work on Design Tensions, offering new ways to view and apply the essential knowledge obtained from the user. This development is described in the context of ongoing work on an exemplar Networked Urban Screens project: Screens in the Wild [1]. This paper will discuss how initial work on Screens in the Wild helped to identify specific user-requirement tensions.

In the early stages of the Screens in the Wild project, the researchers had observed the emergence of such tensions. This started a process of reviewing existing thought on tensions and best practice to resolve them. The process of recognising that tensions may be associated with Networked Urban Screens projects and the review of existing literature is described in early sections of this paper. This is then followed by a key section introducing the proposed Tension Space Analysis. In a later section, the reader is introduced to Screens in the Wild in more depth. Finally, an initial attempt is made to apply Tension Space Analysis to the Screens in the Wild project. 


\section{Networked Urban Screens: A Special Case When Capturing Community Requirements}

During the initial phases of the Screens in the Wild project (which is more fully discussed later in this paper), the research team became increasingly aware of a unique challenge. By connecting four urban screens into a network and embedding this system into several host communities, the importance of capturing, managing and reflecting community requirements (for that screens network) became obvious. In this section, the authors consider the conceptual progression from Urban Screens to Networked Urban Screens. In addition, a brief introduction is provided to the in the wild' approach to conducting research, as used for the Screens in the Wild project. This way of working is particularly relevant to the unique challenge of capturing community requirements for Networked Urban Screens. It is likely that an embedded research team will be exposed to more honest and in-depth feedback from the host communities.

\subsection{Urban Screens}

This section will now consider current research into (non-networked) urban screens. Large urban screens are increasingly becoming a part of the architectural landscape of our cities [2][3]. They are used for advertising, art, local information and global newsfeeds, as well as providing public viewing access to cultural and sporting events [4][5]. In this context it is often proposed that urban screens will somehow help regenerate communities, giving free access to culture and providing a focus for local social cohesion and global networking [6]. However, this technology is not without its problems. A recent report by the Commission for Architecture and the Built Environment $(\mathrm{CABE})$ and English Heritage highlighted concerns for the impact on heritage and urban character, calling for installations to be sensitively designed [5][7]. In other research, issues were found with screens becoming a focus for groups of teenagers and perceived anti social behaviour [8]. Finally, their installation can lead to divided opinions amongst local communities. Previous studies have explored people's social behaviour and relationships to urban screens. For example, Kate Taylor's qualitative studies highlighted behaviours around the BBC screen in Manchester [9]. Many of the large screens in urban environments are stand-alone and offer a nonexistent or very low level of interactivity for the user. Many are 'pushing' information, news or advertising at a passing public who are only invited to respond in a passive, receptive manner. Where networking is considered relevant for installations of this type, it might be used to enable a broadcast media model. For example, in the field of 'digital signage', where the advertisements on multiple large screens may be scheduled and updated remotely [10]. 


\subsection{Networked Urban Screens}

Beyond instances of screen networking for reasons of technical practicality, minimal consideration has so far been given to the creation of screen networks for the purpose of sharing content experiences across communities. Some examples of contentsharing screen networks are briefly described below. Building on the standalone Urban Screens previously described, possibilities for connecting screens are becoming a topic of interest [11]. Such networks offer new possibilities for social interactions and emergent encounters to arise [4][12].

Some projects have suggested using screens to augment the connectivity of remote communities using videoconferencing technologies (eg 'Hole in the Earth' between Rotterdam and Indonesia and the 'Telectroscope' between London and New York). More recently, projects have been exploring connected cities using large urban screens (e.g. Connected Cities with 6 European cities and large screens and the transnational public sphere [13] connecting Melbourne and South Korea). This sense in which cities may be 'connected', is reflected in the multidisciplinary nature of the Screens in the Wild project described in this paper. Networked Urban Screens provide an interesting area not just for HCI researchers, but also for architects, ethnologists and anthropologists, wanting to explore the virtual space created at the liminal interface between the screens [1]. In addition, such multi-disciplinary groups will find interest in the ways that people move through and use the physical spaces around Urban Screens [5][14]. When considering the virtual space that may be created between Networked Urban Screens, it is possible to find useful literature in fields such as virtual environments and mixed reality. For example, researchers have already given consideration to the idea that mixed reality and virtual environments can be used to digitally extend the physical space that people interact within, leading to hybrid-spatial configurations. Consisting of multiple physical and virtual places, these configurations provide a new type of interactional framework. The impact of these on the inhabitant community may require study in the medium to long term [15], [16].

It should be noted that Networked Urban Screens may have multiple modes within which content experiences are shared by the user. Two of the fundamental modes are: synchronous (the content is shared in real-time, similar to an online multi-player action game) or asynchronous (the content is shared 'as and when', similar to social networks. For example: Facebook). Both approaches have advantages and disadvantages. For example, screen content running synchronously is highly dependent on users being available, simultaneously at more than one screen. Conversely, screen content running asynchronously may leave users at each screen unaware that they are part of a screen network and that other users may be interacting concurrently. For this reason, it is important that screen networks (or at least those where the focus is on exploring emergent social behaviour) have methods of continuous real-time interaction, whether the screens are displaying synchronous or asynchronous content.

In the Screens in the Wild project, this is solved through the use of a video link panel, which shows camera feeds from all of the screens (see later section). 


\subsection{Research in the Wild}

The Screens in the Wild project is usefully considered against the background of a specific methodology which underlies this project: research 'in the wild'. This might best be summarized as the development of systems in real world settings. Research 'in the wild' is often described as a process of moving away from the controlled comforts of the research lab and the safety of prototype demonstrations that need only target research colleagues [17][18]. Instead, the research is placed in-situ, throughout all iterations of requirements capture, design, programming and observation. On this, Chamberlain et al. ask: "is it the case that lab-based studies, taking people out of their natural environment and designing in the lab without long term user engagement are no longer appropriate to properly understand the impacts of technology in the real world?" [17].

The relevance of research 'in the wild' in the context of this paper is that: embedding the research team and their developed artefacts into the target community, may lead to a more thorough understanding of how that community functions and how such functionality impacts community expectations in terms of the delivered artefacts. These expectations might relate to the artefact's form, function or meaning. With a researcher's increased awareness of the underlying dynamics in the community, greater insight into the web of conflicting user requirements may emerge. Therefore, research 'in the wild', by nature of its increased focus on the user community and their requirements, is in need of new methods to aid researchers. Tools are required to ensure that maximum benefits are gained from all of the new observational data collected.

\subsection{Tensions}

As the Screens in the Wild research team considered the implications of working 'in the wild' in the relatively unexplored field of Networked Urban Screens, a common theme emerged: 'tensions'. The communities had diverse and often diametrically opposing views concerning the screens network and its possible uses. The team's experience of capturing community requirement tensions will be discussed in more detail later in this paper. Having recognised the presence and importance of these 'tensions', it was decided necessary to further investigate existing ideas on managing requirement tensions in the design process.

\section{Existing work on Design Space and Design Tensions}

When conceptualising and designing a digital artefact or experience, the principles of Human-Centered Design suggest that researchers or practitioners should incorporate the requirements of the end-user community [19][20]. However, the process of requirements capture frequently reveals a complex network of competing values, wants, needs and limitations. The tensions that exist between these requirements may lead to 'design paralysis' [21, p.57]. In this situation, it is not surprising that a common response is to prioritize one or other extreme of a particular tension, 
rejecting the opposite extreme as less important. How such decisions are made, may often lack clarity or rigor. In User Centred Design (a forerunner of work on Design Spaces and Design Tensions), solutions to this problem (moving from design complexity to specification) are usually sought through scientific methods to aid prioritisation [22]. For example weighted values might be applied to requirement attributes, such as: technical feasibility, available resources, time constraints or popularity of a specific requirement within the studied user community.

However, in his discussion on the nature of design practice, Stolterman suggests that scientific methods are not the best tools for handling the complexity of design spaces. He says: "humans try to reduce complexity, to establish control, by making things simpler" [21, p.57]. The complexity surrounding the creation of an artefact or experience is often described as 'the Design Space' [23][24]. Earlier work considers the same phenomenon, under the name Design Rationale. However, the focus of much work on Design Spaces, assumes a need to simplify or filter the tensions, in order to prioritize user requirements. Tatar proposes an alternative analytical framework: 'Design Tensions':

"Design tensions conceptualize design not as problem solving, but as goal balancing. They draw explicit attention to conflicts in system design that cannot be solved, but only handled via compromise" [25, p. 3].

Design tensions "differ from design spaces in that they do not set boundaries or simplify the problem but rather provide a framework for creating a space of relevance." [25, p. 413]. In Design Tensions, Tatar utilizes the older Value Sensitive Design methodology (and how it integrates value considerations) [26].

\section{The Need to Broaden and Extend Existing Work on Design Tensions}

There are clearly some limitations of the existing work on design spaces and tensions and these may now be summarized. With regards to Design Spaces, one issue is that they "are not easily extensible to describe a large number of dimensions or more complex interrelationships" [25, pp. 415-416]. Furthermore, it is assumed with Design Spaces that all requirements will eventually be reduced down to a set of essential artefact attributes. Therefore, some requirements will be labelled as 'less important' to the artefact. On the other hand, it was problematic for us that Design Tensions are often expressed in terms of tension between the user-community and the technology, rather than the requirement tensions within the user-community itself (which we would argue is often the key focus in Networked Urban Screens projects, such as Screens in the Wild). Also, while Design Tensions help us to understand that the simplification of conflicting user requirements may be undesirable, this work still suggests that not all user viewpoints can be reflected in the artefact or experience [25]. 


\section{$5 \quad$ Tension Space Analysis}

In response to the limitations described in the previous section, Tension Space Analysis is offered as a methodology to aid understanding of any tensions that may exist, surrounding the requirements for an artefact.

Extending the existing research on Design Tensions and Design Spaces, Tension Space Analysis focuses on tensions within the user-community itself, not between users and technology. Tension Space Analysis helps to ensure that tensions are represented more fully in the artefact, system or design, rather than being rationalized at the point of design implementation. A visual method of representation for design tensions is provided.

Table 1. Tension Space Analysis step-by-step

Through a variety of ethnographic and requirements capture methodologies,
identify reoccurring tensions within the project or design.
Formalize these tensions in writing.
Consider whether these tensions apply generally, or may be categorized as only
relating to one or more aspects of the project or design (for example system-only,
or screen content experience only).
Visualize the tensions diagrammatically, using a representation that allows for
rapid 'by eye' comparison. Note: at this stage you have a diagram showing the
tensions and their dimensional extremes, but no aspects of the system have
actually been mapped across this representation. This happens in the next stage.
With a radar chart representing the tensions as axial lines, plot on the current
project or design. Use the tension extremes as questions: "is content locally or
globally sourced? Is there any advertising? Etc". Plot on the radar chart
accordingly.
Use the diagrams frequently, to compare system/content versions, when writing
design briefs, when reviewing completed projects and at all stages in a project
lifecycle. Use this as a guide to check that your current thinking reflects all of the
requirement tensions present at the inception of the artefact. If the current artefact
cannot itself practically address all requirements, consider how unrepresented
tensions might be addressed. Can they be represented (or otherwise resolved) in
later versions, updates, sub-elements or complementary artefacts?

The decided visual approach was to look for a relatively simple diagrammatic method to reflect the individual characteristics of a designed artefact (in this case a Networked Urban Screens system and its screen experiences). It was hypothesized that, using a visual method to compare two or more possible design candidates (or built systems, content experiences running on a system etc), would allow distinctive patterns to emerge. As the human eye is adept at perceiving differences between patterns, the intention was to provide a 'quick and dirty' tool to aid iterative design review and comparison. With the user community's requirement tensions always 'present' at meetings (through the diagrams), it becomes harder not to continually address the underlying tensions and to ensure that they remain reflected in ongoing 
design decisions. A 'radar chart' (see the later section, where an example implementation is presented) was chosen for this purpose, as it allows the representation of multiple requirements, each along an axial line, representing its dimensional extrernes. The Tension Space Analysis process is summarized in Table 1 (above).

\section{The Screens in the Wild Project}

Tension Space Analysis emerged from a Networked Urban Screens project called Screens in the Wild. This project considers a specific challenge: how best to integrate urban screens, a radical and potentially disruptive new technology, into the urban realm. As the research for the project is conducted 'in the wild', an essential element has been to establish a network of four urban screens, as a presence in the communities being studied. In fact, the four screens are based in two UK cities, two screens in each city. This network of screens spanning sites in two cities provides the base-platform for the core research of the project. Partners in the project include two universities and local authority / urban regeneration representatives. The entire setup provided a unique opportunity for the research to examine sub-regional interactions (between the communities within the town centres), as well as UK-wide. The Olympics provided an ideal cultural backdrop to the research.

The original research proposal envisaged that the screens would be placed in public areas, such as (the inside of) retail shop windows or accessible public service buildings. Following an active phase of researcher 'outreach' (and in some cases with the assistance of local authority partners), suitable venues were identified for all four screens. All of the screens are positioned inside the front windows of public space venues, visible from the street and/or public areas. The venues are: a public library, a community centre, an art space and a cinema. One aspect of this project's academic study is to consider the screens network from an urban design perspective. Specifically, to develop a theoretical understanding of how the urban experience, mediated through networked urban screens, can be augmented to support communities and culture and the unique design issues related to the implementation of large public screens in urban space [27].

To further this agenda and prior to screen installations, members of the research team studied the built environment in the proposed venue locations. They considered how the urban spaces surrounding the planned screen locations were currently structured and used by different groups of the population. In addition, ethnographic methods were used to capture emergent social interactions in these spaces, before the screens were installed. There then followed a phased installation of all four screens, over a period of several months. During this time, initial screen content prototypes were being built and tested. From the urban design perspective, researchers were interested in the impact of the networked screens on people's relationships with physical, social and interaction space. From the ethnographic perspective, researchers looking at all four networked screen locations (differing in their urban settings and the types of populations they support), were interested in identifying both outcomes that were purely site-specific and those that could be generalized across different sites. As prototype screen content was gradually deployed across the network, a process of 
iterative looping fed back captured user responses and requirements into refined prototypes. The knowledge captured from these iterations, also helped the researchers to generate ideas for potential new screen experiences that might be initiated at a grass roots level by the communities involved. A further strand explored generating ideas for screen content experiences generated by local artists, or artists-inresidence with experience in working with local community groups.

\subsection{The Screen Experiences}

The Screens in the Wild project developed a number of screen experience prototypes. These were implemented on a base system iteratively developed in the first phase of the project. The key aspects of this system (a full description goes beyond the scope of this paper) are as follows. Each 46" screen is mounted vertically (portrait mode) inside a partner venue, i.e. behind glass. A camera and speaker (both available 'offthe-shelf') operate through the glass, making the installation interactive. Each screen node also contains a networked Windows PC, which is remotely administered. The software system uses the UNION Client/Server infrastructure [28] to provide realtime multi-user functionality for web applications across the screen network. Client applications were implemented in HTML5/Javascript to run in a local copy of Firefox full-screen, with scheduler software switching between the experiences described below. To protect the system from undesired remote and local attention R-Kiosk is used in Firefox [29] and Secure Lockdown is used for the desktop [30]. All four nodes are administered remotely using TeamViewer [31].

\section{Video Link}

As general support for interaction and potentially also as a standalone experience, synchronous four-way video communication (no audio) was implemented using a combination of YawCam [32] and iSpy [33]. Both the video panels and the USB camera generating the video feed at each of the nodes have been placed at a low height to encourage interaction from and with children. Currently, video is being displayed as set of four video panes, towards the bottom of the screen (see Fig.1, below). The pane to the left always shows the local feed. It has been mirrored to increase its value as an attractor [34]. The other three panels show video from the remaining three connected screen locations. The Video link encourages synchronous multi-user interaction across the four screen nodes by providing a view of who is interacting and a way to acknowledge or even interact with the other party by waving and through gestures.

\section{SoundShape}

SoundShape provides for collaborative music making between users in different physical locations. It is inspired by the 'Tenori-on' electronic musical instrument, which featured a grid of LED pad switches that could be activated in a number of different ways to create sounds. The visual illumination of active pads produced patterns and displays to complement the music. In SoundShape, this idea is taken a stage further by linking networked urban screens to create a multi-user, networked musical instrument (see Fig.1, left). 

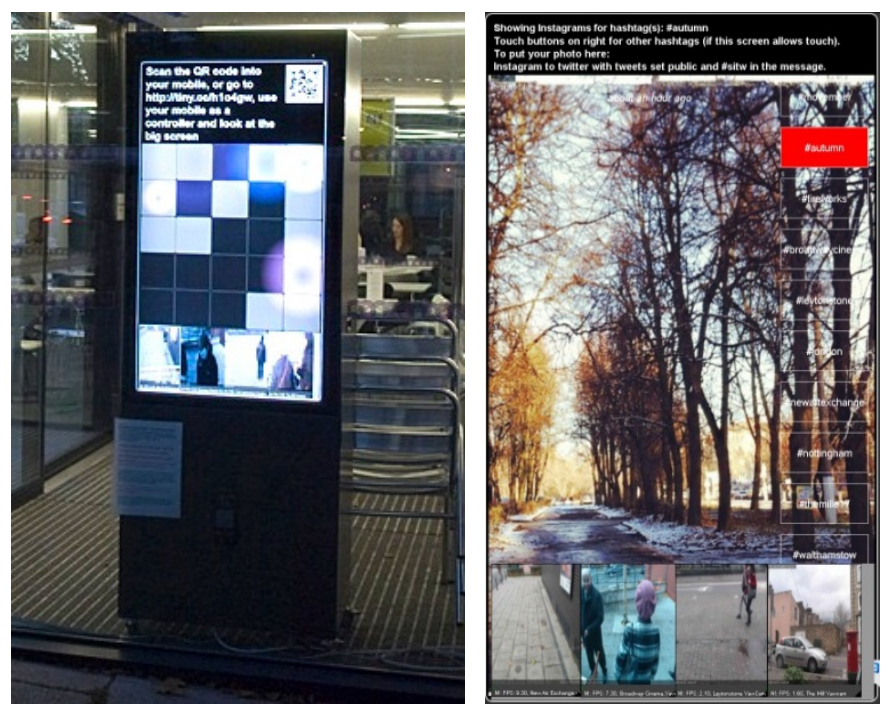

Fig. 1. SoundShape shown in context at one of the venues (left) and a screen shot of ScreenGram (right). The video link is displayed at the bottom of each experience.

Each networked screen has its own SoundShape client. A central server remembers the state of each pad. If a user touches a pad (via touchfoil enabling through-glass touch detection), the server sends this new information to all of the networked screens. All participants see and hear the changes to the musical composition. SoundShape has a $5 \times 5$ grid. Each row represents a note. Each column represents 5 chronological steps in a continually looping musical sequence $(1,2,3,4,5,1$ etc). As notes are added, or removed (by touching the pads to turn them on or off), the musical sequence changes and develops. Active (currently sounding) pads have an animated illumination that flashes in time with the musical sequence. At the one screen where the chosen touchfoil technology does not work with the installed glazing, users can scan an onscreen QR code into their smartphone enabling their phone to work as an interaction device with the screen. SoundShape encourages synchronous multi-user interaction between the four screen nodes. People can share the creation of a single looping music sequence. They can also compete over who can change buttons faster and over which pattern should be on screen.

\section{ScreenGram}

ScreenGram (see above Fig.1, right) searches Twitter for tweets that have specific hashtags, which also have associated Instagram photos. It then retrieves the photos from Instagram and displays them as a constantly updating and revolving slide show. In its simplest form, ScreenGram can display the results for just one hashtag. Users can stand in front of the screen (or anywhere else, but in front of the screen they will get instant feedback), take a photo (or retrieve an image from somewhere else) and send it over Instagram, to Twitter, with the a hashtag of their choice. It usually only takes from 30 seconds to a minute to retrieve a new photo that has been tagged and 
for it to appear on the networked screen. The buttons on the right-hand side of the screen allow the user to display the results for other (currently relevant) hashtags. For example, the user can also choose to show the latest photos for: \#summer, \#olympics etc. It is very easy for the researchers (or in theory the community, venue staff, etc.) to change the hashtags that appear on these buttons. The buttons are 'radio buttons'. So, clicking one will disable any already selected and each button toggles on (red) and off (clear). ScreenGram encourages asynchronous multi-user interaction between the networked screens and people elsewhere interacting through their phones. They can actively participate by posting images to the screen, and for this interaction they do not have to be at the screen location. At any of the nodes, people can also filter the onscreen content and watch the slideshow that is determined by which hashtag is currently selected.

\section{Capturing Community Requirement Tensions in the Screens in the Wild Project}

Before, during and after the initial screen content prototypes were in development, researchers from the Screens in the Wild project observed the communities located near to the venues. It is in the nature of research 'in the wild' that the distinctions between user, researcher and any other person in contact with an artefact become blurred. Certainly the role of the researcher as a dispassionate, objective 'gatekeeper' is highly questionable, when he or she is embedded into the community under study. In a sense, the researcher becomes a part of an 'ecology', shared with the local community and anyone else that interacts with or is in any way impacted by the presence of the introduced artefact. Some of the methods used included: observations, conversations, interviews and workshops.

Researchers conducted semi-structured interviews with managers of the organizations that allowed us to install a screen node in their premises. The purpose of these interviews was to better understand their perception or perspective of the project. Before the development and implementation, the researchers organised four workshops with members of the public at two locations in London. The intention of these workshops was varied. In some cases, the goal was to analyze the usability, in others to enable some production of local content for the screen, but in general the research team was seeking to receive feedback or suggestions of different kinds on the work completed so far.

In order to kickstart the development of screen content prototypes, the academic partners held a bodystorming session in a research lab. Similar to brainstorming, bodystorming encourages participants to move around, use their bodies and to use physical or paper mockups as stand-ins for the proposed artefact(s). In this case, functioning prototypes of the hardware and software system were set up in the research lab. Participants were requested to test what was built so far and suggest new ideas for development. Later on in the project, the stakeholders of the first venue to deploy at were invited to a similar session to give them a sense of what would be installed later in the project. 
Researchers also participated in using the screen content prototypes, alongside communities, uploading their own images onto the screen nodes, sharing their perspectives and personal views.

Later, the team reflected on its own participation, producing field notes and summaries of their screen interaction sessions. This enabled the researchers to understand the context of each screen experience in a more profound way. The team's time spent as 'co-participants' better prepared them to respond to any experiencerelated concerns expressed by the user-community participants. Researchers conducted sixty-four structured interviews with people belonging to different social groups and communities in neighbourhoods where the screens are located. The people interviewed had a diverse level of engagement with the social life of the neighbourhoods.

\subsection{Identified Tensions in Community Requirements}

As the Screens in the Wild project still has several months left to run, formal evaluation has not been completed. That said, the conducted observations, interviews and workshops have already thrown up a fascinating raw dataset. This section describes the process of distilling the unstructured community feedback, down to a series of observed tensions.

Table 2. Sample opinions from the raw dataset

\begin{tabular}{|c|}
\hline rns over \\
\hline $\begin{array}{l}\text { "People are starting to get the hang of the schedule, especially the lights. Some } \\
\text { comments on the schedule from the public: 'Can you turn the game on now?', 'Can } \\
\text { you show me that picture from this morning?' suggest that people think we're in } \\
\text { control of the schedule, and indeed that we control the screen as a whole." }\end{array}$ \\
\hline $\begin{array}{l}\text { On the Sound Shape experience "The lights game: this is the most popular app, } \\
\text { with users of all ages, but especially appealing to pre-teen children. Some adults } \\
\text { have asked what the point of the game is, but kids largely make up their own rules } \\
\text { and get quite competitive. It's not unusual to see a group of two or three children } \\
\text { playing with the squares all at once, with an adult looking bemused over their } \\
\text { shoulder." }\end{array}$ \\
\hline $\begin{array}{l}\text { Participants emphasized the importance of having an open system, where anyone } \\
\text { could upload pictures, curate and engage with the screens in personal terms. This } \\
\text { involved less censorship from institutions and more user options for } \\
\text { choosing/producing what they would like to see. }\end{array}$ \\
\hline $\begin{array}{l}\text { People revealed that they ignored public screens present at nearby local shopping } \\
\text { centres or other public venues. They have learned to dismiss them as part of } \\
\text { annoying highly-commercial advertising. }\end{array}$ \\
\hline
\end{tabular}

Table 2. (above) presents some sample opinions (both formal and informal) expressed by the user communities and venue owners/managers. 
In the team meetings and discussions following the most recent round of observations, it started to become obvious that certain requirements (or 'what they would like to see the screen network displaying in their community' to put it more simply) were repeatedly expressed by the local communities. What struck the researchers was how frequently there was complete polarization of opinion on a single issue. For example, person1 says: "the last thing I'd want to see is advertising keep it non-commercial" vs. person2 says, "it would be great for advertising local businesses". Having recognized the existence of these 'tensions', it was interesting to record and consider them. Table 3. presents the observed dimensions of tension, labeled in natural language, as they had expressed in interviews and conversations.

Table 3. Identified requirement tensions

\begin{tabular}{|l|l|l|}
\hline Moderated community & vs. & Open community \\
\hline Auto scheduled & vs. & Community/user scheduled \\
\hline $\begin{array}{l}\text { Cities hosting screens too culturally } \\
\text { similar, contrast not great } \\
\text { enough...screens should be more } \\
\text { geographically/culturally separated }\end{array}$ & vs. & Don't know where cities are! \\
\hline More local content & vs. & More generalised content \\
\hline Local advertising & vs. & Non-commercial \\
\hline Content not personal enough & vs. & Content too personal \\
\hline $\begin{array}{l}\text { Content with history, places, things } \\
\text { and tangible objects important - } \\
\text { stimulate memories }\end{array}$ & vs. & $\begin{array}{l}\text { People, relationships important, } \\
\text { current moment important - } \\
\text { don't care about things }\end{array}$ \\
\hline
\end{tabular}

It is important to stress that these are only some of the possible tensions that might exist within a project. They may be unique to the Screens in the Wild project and no claim is made to have identified a comprehensive set of tensions. There may well be some tensions that are common to all projects/artefacts/experiences, but it is assumed that each situation will generate its own unique 'tension space'. In addition, tensions may exist across many sub-domains of the project (for example: community vs. technical, community vs. researchers, venue owners vs. venue users etc). At this stage, attention is only being given to tensions that exist within the user community itself (venue users, venue owners, local residents etc), as these are the groups physically impacted by the introduction of a screen network, into their local urban space.

Next, some of these natural language tension descriptions were translated into shorter more descriptive labels (partially for easier use in visual representations):

- moderated community vs. open community (unchanged): can content be added to the system (or individual screen experience, if applied to this) by the public, or is there some level of moderation? 
- auto scheduled vs. community/user scheduled (unchanged): is the programming of the screen content (time of day, choice of experience etc) controlled by the researchers automatically (using a scheduler application) or in the hands of the venues or venue users?

- locations sufficiently different vs. locations not different enough (formerly: Cities hosting screens too culturally similar, contrast not great enough...screens should be more geographically/culturally separated vs. Don't know where cities are!): some users thought that the cities hosting the screens were too similar, others (from one city), did not know the location of the second city.

- locally sourced vs. globally sourced (formerly: More local content vs. More generalised content): is screen content created in the community, or could it come from anywhere (over the Internet)? Is this content about this community (or this screens network), or is it location-neutral?

- (local) advertising vs. no advertising (formerly: Local advertising vs. Noncommercial): should the network (or experience) be commercial or completely non-commercial?

- personal vs. non-personal (Content not personal enough vs. Content too personal): some users were very worried about sharing personal information. Where was it going? Who would see it? Others, wanted experiences to be more personal.

- things \& past vs. people \& now (formerly: Content with history, places, things and tangible objects important - stimulate memories vs. People, relationships important, current moment important - don't care about things): there was a noticeable gap between users wanting to see images of past times and events (often coupled with low levels of interactivity from them) and users wanting to see current (often social) content. Sometimes, this division reflected a difference between age groups, but not always.

\section{Applying Tensions Space Analysis to the Screens in the Wild Project}

Now, it is possible to apply the Tension Space Analysis concepts developed thus far to current elements of the Screens in the Wild project. Referring back to the tensions identified earlier in the paper, some of them are immediately more relevant to the baseline screen network system itself (without screen experiences), whereas other tensions are relevant to the screen content experiences.

The figures below, illustrate the mapping of the identified the tensions, as dimensions on two separate radar charts. Fig. 2. shows the dimensions primarily relevant to the baseline screen network. Fig. 3. presents the dimensions considered more relevant to the screen experiences. These radar axial radar charts may be regarded as 'empty templates', ready for the assessment of the system and screen experiences relating to the studied artefact.

Referring back to Table 1, once the empty axial line radar charts have been created, the next step is to superimpose the current project or design. To achieve this, it may 


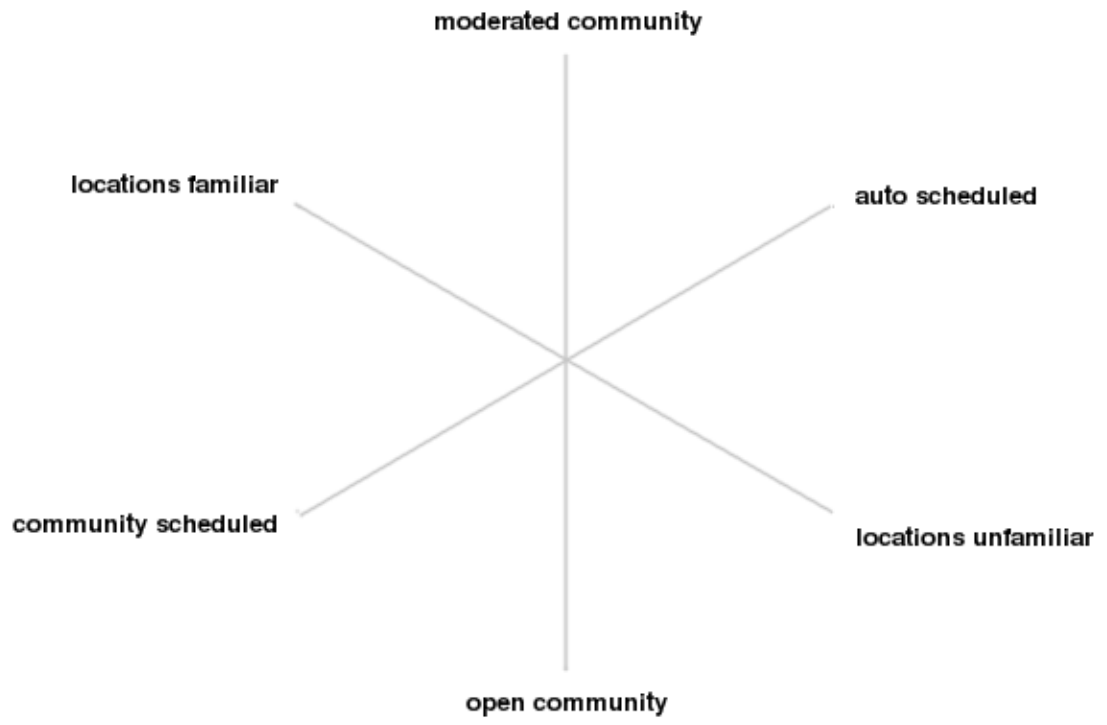

Fig. 2. The system-related tensions shown as dimensions on a radar chart

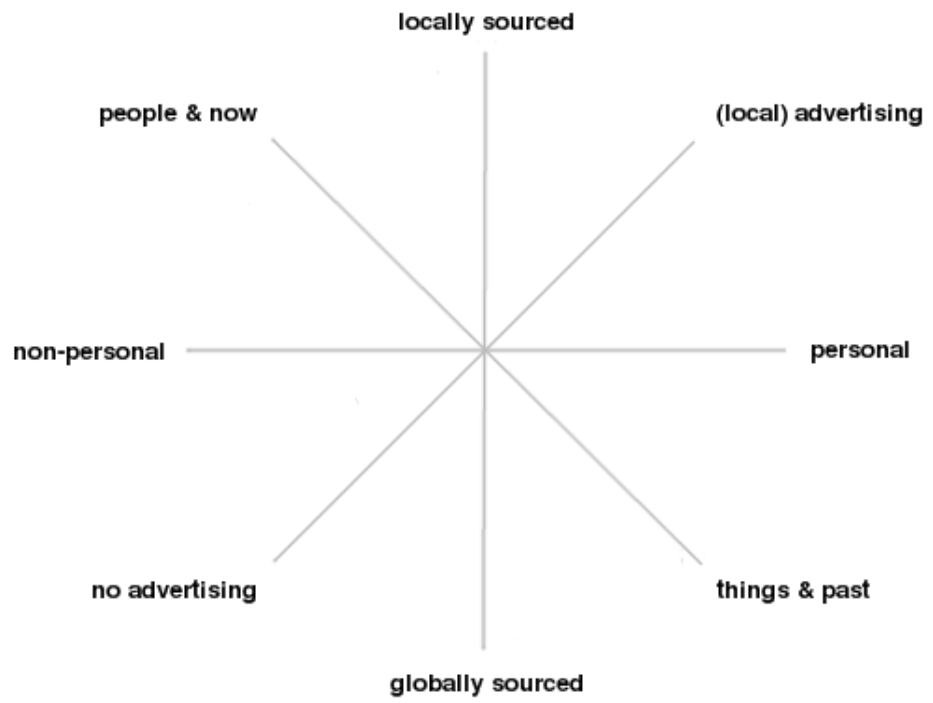

Fig. 3. The experience-related tensions shown as dimensions on a radar chart

be useful to consider the 'tension extremes' on each axial line in terms of questions (for yourself or the project team): "is content locally or globally sourced?", "is there any advertising?" etc. The results of this are then plotted onto the radar chart accordingly. The figures below show the tensions applied to both the baseline system (see Fig. 4.) and an example screen experience - ScreenGram (see Fig. 5.). 


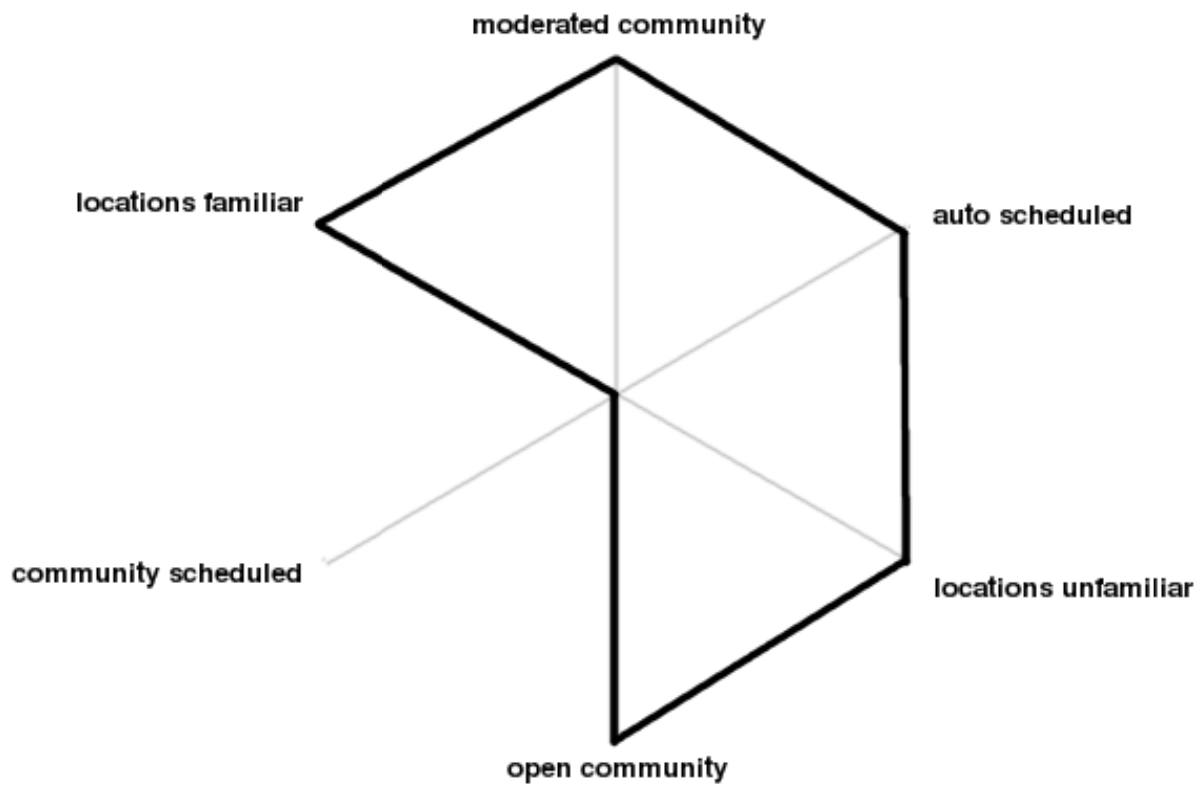

Fig. 4. Tension Space Analysis radar chart for the current screen network system

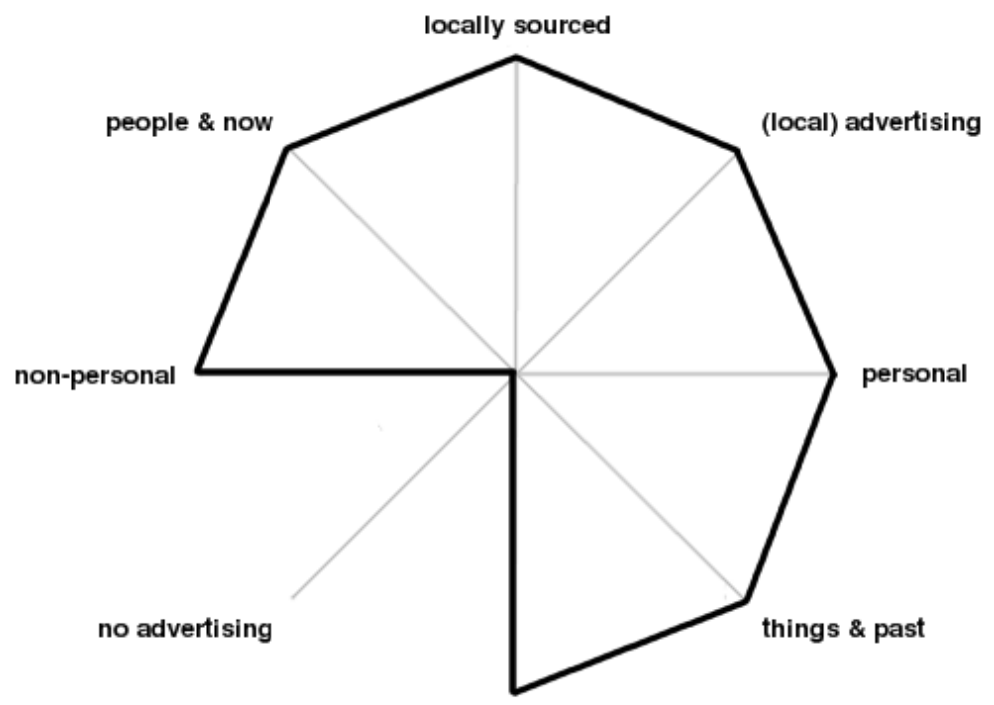

globally sourced

Fig. 5. Tension Space Analysis radar charts for the ScreenGram prototype experience 
Looking at the radar chart for the current screen network system (see Fig. 4.), we can see that it is capable of hosting both open and moderated communities (dependent on the experience running), that content is automatically scheduled, that users have reported both that the cities hosting the screens are culturally familiar (often implying that more distinction would be preferable) and also that one of the other host cities is unfamiliar to them (often implying that they regard the differences as sufficient to make the system interesting to them).

Looking at the radar chart for the ScreenGram prototype experience (see Fig. 5.), it can be seen that most of the tensions are represented - a pleasing result!. The highlighting on the advertising tension line shows that users desiring 'no advertising' may be disappointed. This is because ScreenGram's content is open (it represents an example of the 'open community' axial extreme, shown as active for the entire screen network system - see Fig. 4.). Anyone can post an image to the screen network by applying a specific hashtag. Therefore, it is possible advertising might appear and the 'no advertising' end of the axial line is not highlighted.

\section{Conclusions}

Drawing on a live, 'in the wild' research project (Screens in the Wild), we have identified some of the design tensions that might be encountered when designing any network of urban screens (for both the system itself and its screen content experiences). These tensions are likely to emerge because of the different views that multiple stakeholders (located within very different communities) have about the use of such a screen network.

Tension Space Analysis proposes an approach for capturing and visualizing existing user requirements and ensuring that they are reflected in the design, or operational modes of the final artefact or experience. In Tension Space Analysis we would suggest that it is essential to keep alive an essence of all user requirement tensions within the artefact, even if particular aspects of each dimension are emphasized either in the final static artefact, or through different aspects/modes of the artefact's function. For example, in the field of Networked Urban Screens, each screen experience that is displayed across the screens offers an opportunity to reflect some of the tensions.

Therefore, it is not necessarily the main goal to reflect all of the tensions in the totality of the system. All tensions might be incorporated gradually over its lifetime. In addition, Tension Space Analysis specifically considers tensions within the requirements of user-communities, as opposed to Design Tensions, which primarily focuses on user community versus technology tensions.

The contribution made by Tension Space Analysis is to broaden and extend existing thought on Design Tensions, to include new domains and methods of representation. Specifically, Design Tensions are improved in two specific ways.

Firstly, the focus of analysis is shifted from tensions that exist between users and technology (as in Design Tensions), to the tensions that exist within the user community itself. Secondly, Tension Space Analysis introduces a visual aid to 
understanding user-requirement tensions. This will then be used at all stages in the project to discourage researchers/designers from prematurely discarding tensions before fully considering how they might best be represented in the artefact itself, either in the short-term or during its entire lifecycle.

With an increasing HCI focus on 'in the wild' research and user community engagement, there is a pressing need for approaches such as Tension Space Analysis. It is hoped that using techniques such as this will help researchers to document and then frequently revisit the full spectrum of community requirements, ensuring maximum representation of the target community within a delivered artefact.

Acknowledgements. We would like to gratefully acknowledge the support from the Engineering and Physical Sciences Research Council (EPSRC) through grant EP/I031413/1 (Exploring the potential of networked urban screens for communities and culture) as well as the continued support from our partner venues.

\section{References}

1. Screens In The Wild, http://www. screensinthewild.org/

2. Brignull, H., Izadi, S., Fitzpatrick, G., Rogers, Y., Rodden, T.: The introduction of a shared interactive surface into a communal space. In: Proceedings of the 2004 ACM Conference on Computer Supported Cooperative Work, pp. 49-58 (2004)

3. Struppek, M.: The social potential of Urban Screens. Visual Communication 5, 173 (2006)

4. Fatah gen Schieck, A.: Towards an integrated architectural media space. First Monday 4 (2006)

5. Fatah gen Schieck, A., Boddington, G., Fink, P.: Framework for the implementation of urban big screens in the public space (2009)

6. McQuire, D.S.: The media city: Media, architecture and urban space. Sage Publications Ltd. (2008)

7. Large digital screens in public spaces. Joint guidance by English Heritage and CABE (2009)

8. Fatah gen Schieck, A.: A tale of two cities. In: Urban Screens 2008, Melbourne, Australia (2008)

9. Taylor, K.: Programming video art for urban screens in public space. First Monday (2006)

10. Memarovic, N., Langheinrich, M., Alt, F.: Interacting Places - A Framework for Promoting Community Interaction and Place Awareness through Public Displays. In: Adjunct Proceedings of the Tenth Annual IEEE International Conference on Pervasive Computing and Communications (2012)

11. Davies, N., Langheinrich, M., José, R., Schmidt, A.: Open Display Networks: A Communications Medium for the 21st Century. IEEE Computer 45(5), 58-64 (2012); Special Issue on Interactive Digital Signage

12. Memarovic, N., Langheinrich, M., Alt, F.: The Interacting Places Framework Conceptualizing Public Display Applications that Promote Community Interaction and Place Awareness. In: Proceedings of the International Symposium on Pervasive Displays (PerDis 2012). ACM, New York (2012)

13. Papastergiadis, N.: Spatial Aesthetics: Rethinking the Contemporary. In: Smith, E., Condee (eds.) Antinomies of Art and Culture, pogl., vol. 19 
14. Brynskov, M., Dalsgaard, P., Ebsen, T., Fritsch, J., Halskov, K., Nielsen, R.: Staging urban interactions with media façades. In: Gross, T., Gulliksen, J., Kotzé, P., Oestreicher, L., Palanque, P., Prates, R.O., Winckler, M. (eds.) INTERACT 2009. LNCS, vol. 5726, pp. 154-167. Springer, Heidelberg (2009)

15. Schnädelbach, H., Penn, A., Steadman, P., Benford, S., Koleva, B., Rodden, T.: Moving office: inhabiting a dynamic building. In: Proceedings of the 2006 20th Anniversary Conference on Computer Supported Cooperative Work, pp. 313-322 (2006)

16. Schnädelbach, H.: Hybrid spatial topologies. The Journal of Space Syntax 3(2), 204-222 (2012)

17. Chamberlain, A., Crabtree, A., Rodden, T., Jones, M., Rogers, Y.: Research in the wild: understanding 'in the wild' approaches to design and development. In: Proceedings of the Designing Interactive Systems Conference (DIS 2012), pp. 795-796. ACM, New York (2012)

18. Rogers, Y.: Interaction design gone wild: striving for wild theory. Interactions 18(4), 58$62(2011)$

19. Chayutsahakij, P.: Human Centered Design Innovation. Institute of Design. IIT Technology (2000)

20. Vanden Abeele, V.A., Van Rompaey, V.: Introducing human-centered research to game design: designing game concepts for and with senior citizens. In: Vanden Abeele, V.A., Van Rompaey, V. (eds.) CHI 2006 Extended Abstracts on Human Factors in Computing Systems (CHI EA 2006), pp. 1469-1474. ACM, New York (2006)

21. Stolterman, E.: The nature of design practice and implications for interaction design research. International Journal of Design 2, 55-65 (2008)

22. Garrett, J.J.: The Scope Plane: Functional Specifications and Content Requirements. The Elements of User Experience: User-Centered Design for the Web. In: Prioritizing Requirements, ch. 4. Peachpit Press (2002)

23. Card, S.K., Mackinlay, J.D., Robertson, G.G.: The design space of input devices. In: Proceedings of the SIGCHI Conference on Human Factors in Computing Systems: Empowering People, pp. 117-124 (1990)

24. MacLean, A., Young, R., Bellotti, V., Moran, T.: Questions, Options, and Criteria: Elements of Design Space Analysis. Human-Computer Interaction 6(3\&4), 201-250 (1991); Special Issue on Design Rationale: Carroll, J.M., Moran, T.P. (eds.)

25. Tatar, D.: The design tensions framework. Human-Computer Interaction 22, 413-451 (2007)

26. Friedman, B.: Value-sensitive design. Interactions 3, 16-23 (1996)

27. Fatah gen Schieck, A., Schnädelbach, H., Penn, A.: Research in the Wild: Exploring the potential of Networked Urban Screens for Communities and Culture. In: Research in the Wild Workshop, DIS 2012, Newcastle, UK (2012)

28. Union Platform, By USER1, http: / / www . unionplat form. com

29. R-kiosk Firefox plugin, https : / / addons . mozilla.org/ en-us/firefox/addon/r-kiosk/

30. Secure Lockdown, http: / / shop. inteset. com/Products / 9-lock-down-windows-7-with-inteset-secure-lockdown. aspx

31. TeamViewer, http://www. teamviewer.com

32. YawCam, http://www. yawcam.com

33. iSpy, http: //www. ispyconnect.com

34. Müller, J., Walter, R., Bailly, G., Nischt, M., Alt, F.: Looking glass: a field study on noticing interactivity of a shop window. In: Proceedings of the SIGCHI Conference on Human Factors in Computing Systems, pp. 297-306. ACM, New York (2012) 